\title{
"Walking into the unknown..." key challenges of pregnancy and early parenting with inflammatory arthritis: a systematic review and thematic synthesis of qualitative studies
}

Nevena Rebic ${ }^{1,2,3}$, Ria Garg ${ }^{1,3}$, Ursula Ellis ${ }^{4}$, Vanessa Kitchin ${ }^{4}$, Sarah Munro ${ }^{5,6}$, Glen Hazlewood ${ }^{2,7}$, Neda Amiri ${ }^{8}$, Nick Bansback ${ }^{6,9}$ and Mary A. De Vera ${ }^{1,2,3^{*}}$

\begin{abstract}
Background: To conduct a systematic review and thematic synthesis of qualitative studies on the pregnancy and early parenting experiences of patients with inflammatory arthritis (IA).

Methods: We searched online databases for English-language, qualitative studies capturing the experiences of females with IA or their healthcare providers with pregnancy and/or early parenthood. We extracted findings from included studies and used thematic synthesis to develop descriptive and higher-order analytical themes.

Results: Of 20 included studies, our analysis identified 5 analytical themes among patients and 3 among providers. Patients' reproductive desires, the impact of IA on their ability to experience pregnancy, and the availability of information to guide preparedness informed their pregnancy decisions. Patients' IA management, pregnancy expectations, and access to support influenced their reproductive experiences. Patients' experiences seeking information and care revealed substantial gaps in reproductive care provision to patients with IA. Reproductive uncertainty related to IA placed a heavy burden on patients' emotional and psychological wellbeing. Reproductive care provision was influenced by providers' perceived professional responsibility to address patients' reproductive goals, fears of negative outcomes, and capacity to harness patient trust, incorporate reproductive care into rheumatology practice and facilitate multi-disciplinary care coordination.
\end{abstract}

Conclusions: Our review illuminated several barriers to experiencing pregnancy among patients with IA, particularly related to pregnancy planning support, availability of information, and care coordination among the patient's healthcare team. To improve care, these barriers may be mitigated through the provision of relevant, practical, and consistent information as well as patient-centred multi-disciplinary approaches for managing pregnancy among patients with IA.

Keywords: Inflammatory arthritis, Pregnancy, Systematic review, Qualitative methods

\footnotetext{
* Correspondence: mdevera@mail.ubc.ca

${ }^{1}$ Faculty of Pharmaceutical Sciences, University of British Columbia, 2405

Wesbrook Mall, Vancouver, BC V6T 1Z3, Canada

${ }^{2}$ Arthritis Research Centre of Canada, 5591 Number 3 Rd, Richmond, BC V6X

2C7, Canada

Full list of author information is available at the end of the article
}

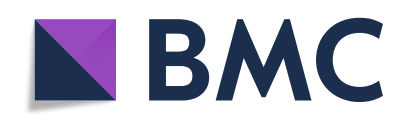

(- The Author(s). 2021 Open Access This article is licensed under a Creative Commons Attribution 4.0 International License, which permits use, sharing, adaptation, distribution and reproduction in any medium or format, as long as you give appropriate credit to the original author(s) and the source, provide a link to the Creative Commons licence, and indicate if changes were made. The images or other third party material in this article are included in the article's Creative Commons licence, unless indicated otherwise in a credit line to the material. If material is not included in the article's Creative Commons licence and your intended use is not permitted by statutory regulation or exceeds the permitted use, you will need to obtain permission directly from the copyright holder. To view a copy of this licence, visit http://creativecommons.org/licenses/by/4.0/. The Creative Commons Public Domain Dedication waiver (http://creativecommons.org/publicdomain/zero/1.0/) applies to the data made available in this article, unless otherwise stated in a credit line to the data. 


\section{Background}

Inflammatory arthritides (IA), including rheumatoid arthritis (RA) and systematic lupus erythematosus (SLE), are chronic inflammatory diseases that commonly affect female patients of reproductive age [1, 2]. As disease activity and associated medication use may cause pregnancy complications, patients with IA face unique challenges related to planning and managing pregnancy [2].

The reproductive health needs of individuals with IA are often inadequately managed. In a 2012 international survey of female patients with chronic inflammatory conditions, including IA, the majority of respondents reported pregnancy was not sufficiently addressed in clinical practice and only $40 \%$ received consistent reproductive guidance from healthcare providers [3]. Indeed, there is a body of research indicating patients have substantial information needs about pregnancy and early parenting, particularly related to medication use [4-6]. Poor dissemination of medication information for pregnancy can lead patients to discontinue beneficial medications due to fear of foetal harm, which may result in poorer outcomes for both mother and baby related to active disease [7]. Given the complexity of managing pregnancy with IA, we aimed to systematically review the qualitative literature reporting patient and provider perspectives of reproductive care provision for female patients with IA.

\section{Methods}

\section{Search strategy}

We developed and refined our search strategy parameters in consultation with two health sciences librarians (UE, VK). We searched Ovid MEDLINE, Ovid EMBASE, CINAHL, PsycINFO via EBSCOHost, and Social Sciences Citation Index from inception to February 2020. Additionally, we conducted a hand search of relevant bibliographies. We mapped subject headings and keywords of unindexed terms related to IA, pregnancy and parenthood, and qualitative methods (see Additional file 1) to identify citations meeting our inclusion criteria: primary research article; study sample of female patients with IA (i.e., RA, SLE, ankylosing spondylitis [AS], juvenile idiopathic arthritis [JIA], psoriatic arthritis [PsA]) or their healthcare providers, qualitative study design capturing participants' pregnancy and/or early parenthood experiences, and published in English. The enhancing transparency in reporting the synthesis of qualitative research (ENTR EQ) statement guided our reporting [8].

\section{Study selection}

Two authors (NR, RG) independently reviewed titles and abstracts of identified records. Studies meeting the inclusion criteria received full text review. Thereafter, those that continued to satisfy inclusion criteria were forwarded for quality assessment and systematic review. Disagreement was resolved by consensus. We extracted information on the publication year, country, diseases studied, participant characteristics (i.e,. age, sex, patient/provider), study objective, data collection, and analysis methods, reported reproductive topics.

\section{Quality assessment}

We used the Critical Appraisal Skills Programme (CASP) Qualitative Research Checklist $[9,10]$ to assess the quality of included studies. This checklist comprises 10 questions assessing research aims, design, methodology, recruitment, data collection, data analysis, ethical issues, participant-researcher relationship, findings, and research value [9]. Two authors (NR, RG) independently evaluated studies and resolved disagreements through discussion.

\section{Synthesis}

Included studies were imported into NVivo 12.6.0. We analysed study results data, including primary quotations and authors' interpretations included in the text, tables, and supplementary materials. One author (NR) preformed the thematic synthesis, a method combining grounded theory and meta-ethnography approaches $[11,12]$. This involved conducting line-by-line coding to develop an initial coding framework which was subsequently applied across all articles [11]. Similarities and differences between codes were used to inform their hierarchical grouping into descriptive themes [11]. Relationships within and between descriptive themes were analysed to generate analytical themes of higher-order constructs that addressed our review objectives [11]. Two authors (NR, MDV) reviewed the coding framework and discussed developing themes.

\section{Results}

Our search (Fig. 1) identified 1347 articles. After exclusions, we included 20 studies for thematic analysis (Table 1$)$. The majority $(n=19)$ reported on 368 female patient perspectives and 3 studies reported on 51 provider perspectives. With respect to disease, 14 studies limited inclusion to a single diagnosis (8 examined RA patients, 4 SLE, and 2 JIA) with remaining studies including a mix of IA patients. Studies reporting provider perspectives compromised primarily rheumatologists as well as midwives, general practitioners, nephrologists, and health visitors.

The CASP Checklist appraisal is summarized in Table 2. All studies clearly stated their aims, appropriately used a qualitative research methodology, and adequately justified its use. Most studies collected, analysed, and reported data adequately, used rigorous data analysis, and clearly stated research findings. 


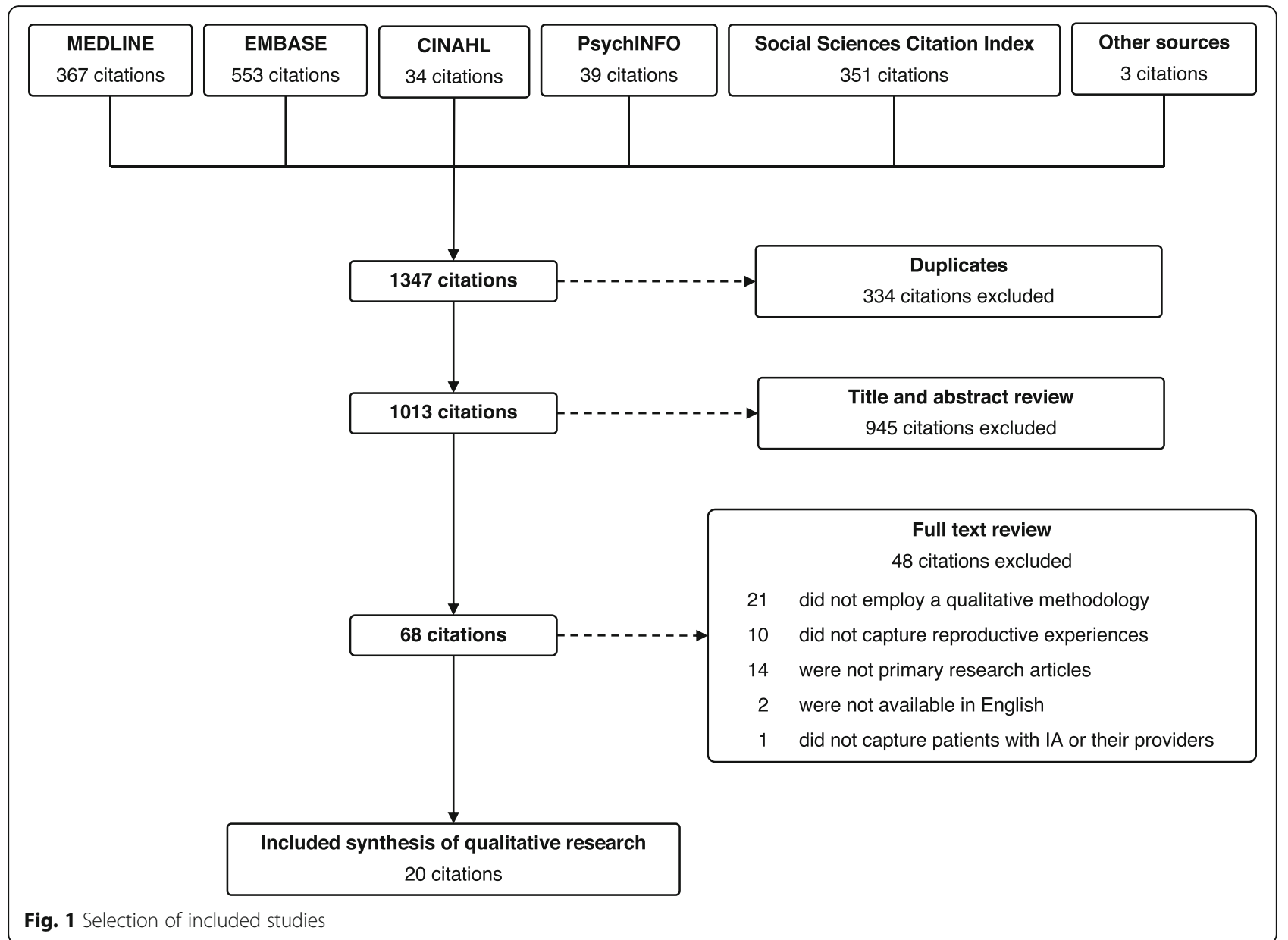

Our synthesis identified 5 analytical themes among patients: making decisions about planning and experiencing pregnancy, experiencing pregnancy and parenting, navigating caregiving with chronic disease, seeking information and resources for pregnancy planning, and interacting with healthcare providers. We additionally identified 3 analytical themes among providers: providing reproductive health care, interacting with patients, and coordinating patient care with other providers. Our coding framework and representative quotations are presented in Table 3 . Relationships between analytical and descriptive themes are depicted in a conceptional model (Fig. 2).

\section{Patient perspectives}

\section{Making decisions about planning and experiencing} pregnancy

Making decisions about having children comprised a dynamic balance of patients' reproductive desires, the impact of disease on their ability to experience pregnancy, and the availability of information to guide preparedness. We identified 5 descriptive themes related to patients' reproductive decision-making in 17 studies $[4,5,13,14$, $16,17,19-24,26-30]$.
When discussing making decisions about becoming pregnant, patients emphasized the role of their disease within this process $[5,13,16,17,19-24,26,29,30]$. Many expressed concerns about disease activity and management during pregnancy and post-partum $[5,24$, $29,30]$ and shared fears of being unable to care for a child due to their IA [20, 21, 24, 29, 30]. Some patients expressed strong desires to bear children despite these concerns $[20,26,29,30]$ or sought information about alternatives to childbearing (e.g., adoption) $[5,16,29]$. Others chose not to have children or limited their family size $[17,18,20,21,23,24,29,30]$. Factors influencing childbearing decisions included their medications $[5,17$, $20,22,29]$, disease severity $[5,17,20,30]$, previous pregnancy experiences $[20,30]$, and support from partners, family, and healthcare providers $[5,19,20,30]$. Patients in one study discussed navigating contraception planning and pregnancy termination [30].

Making decisions about using medications [4, 5, 13, 14, 20, 22, 26, 27, 29] and breastfeeding [5, 20] were closely intertwined with reproductive decisions. Patients reported desiring more information about medication safety related to fertility, pregnancy, and breastfeeding $[4,5,20,29]$, 


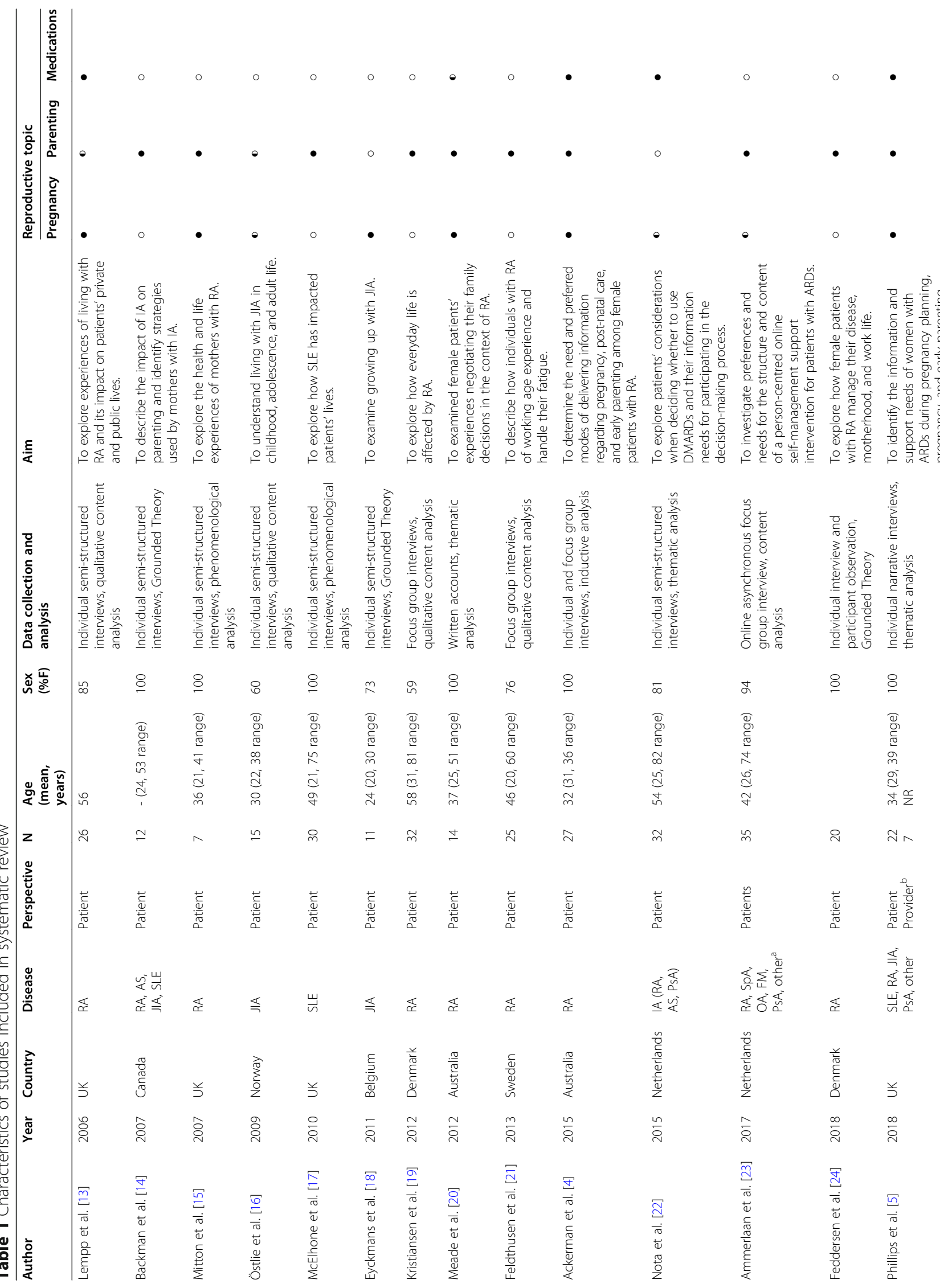




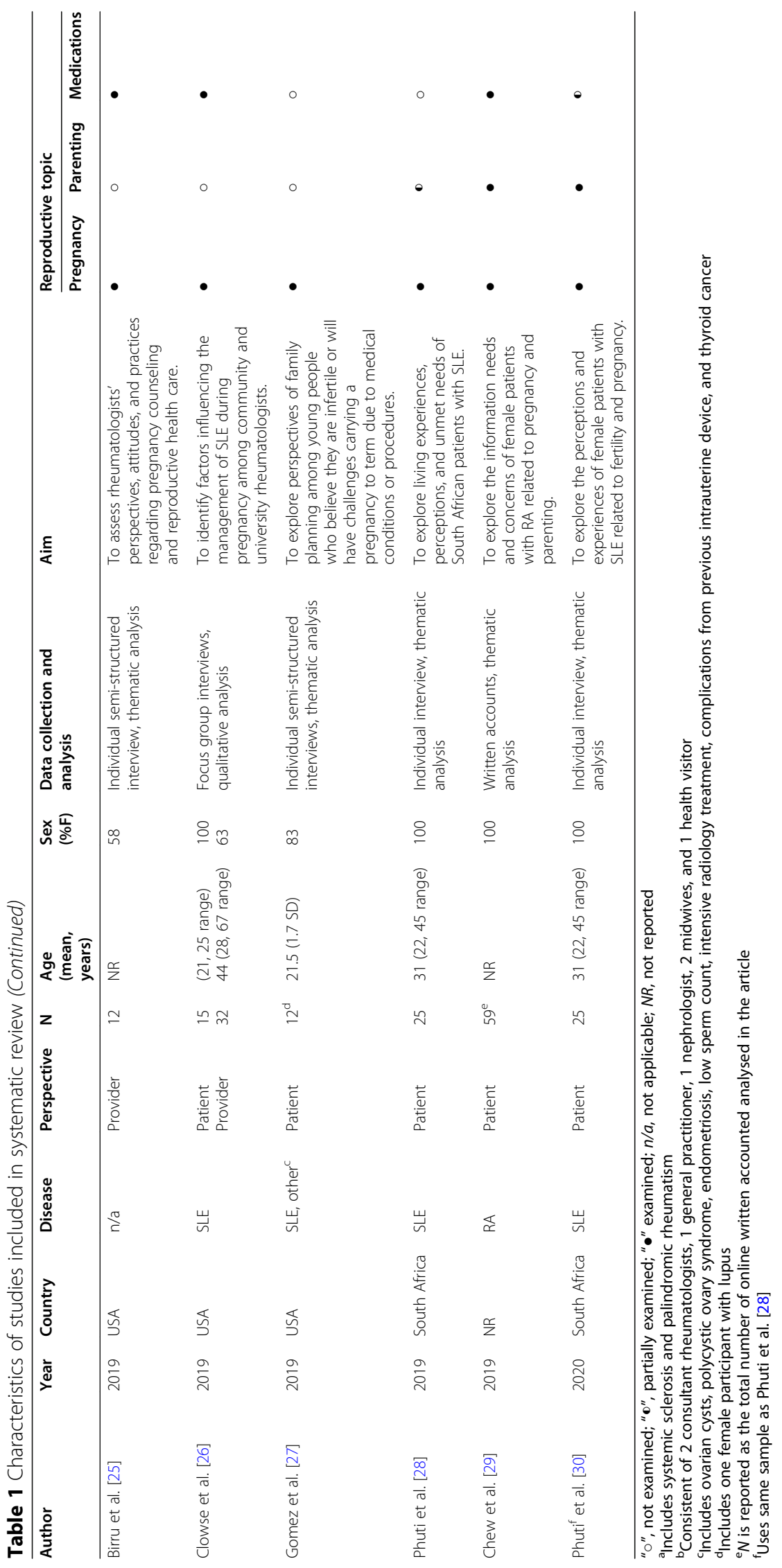




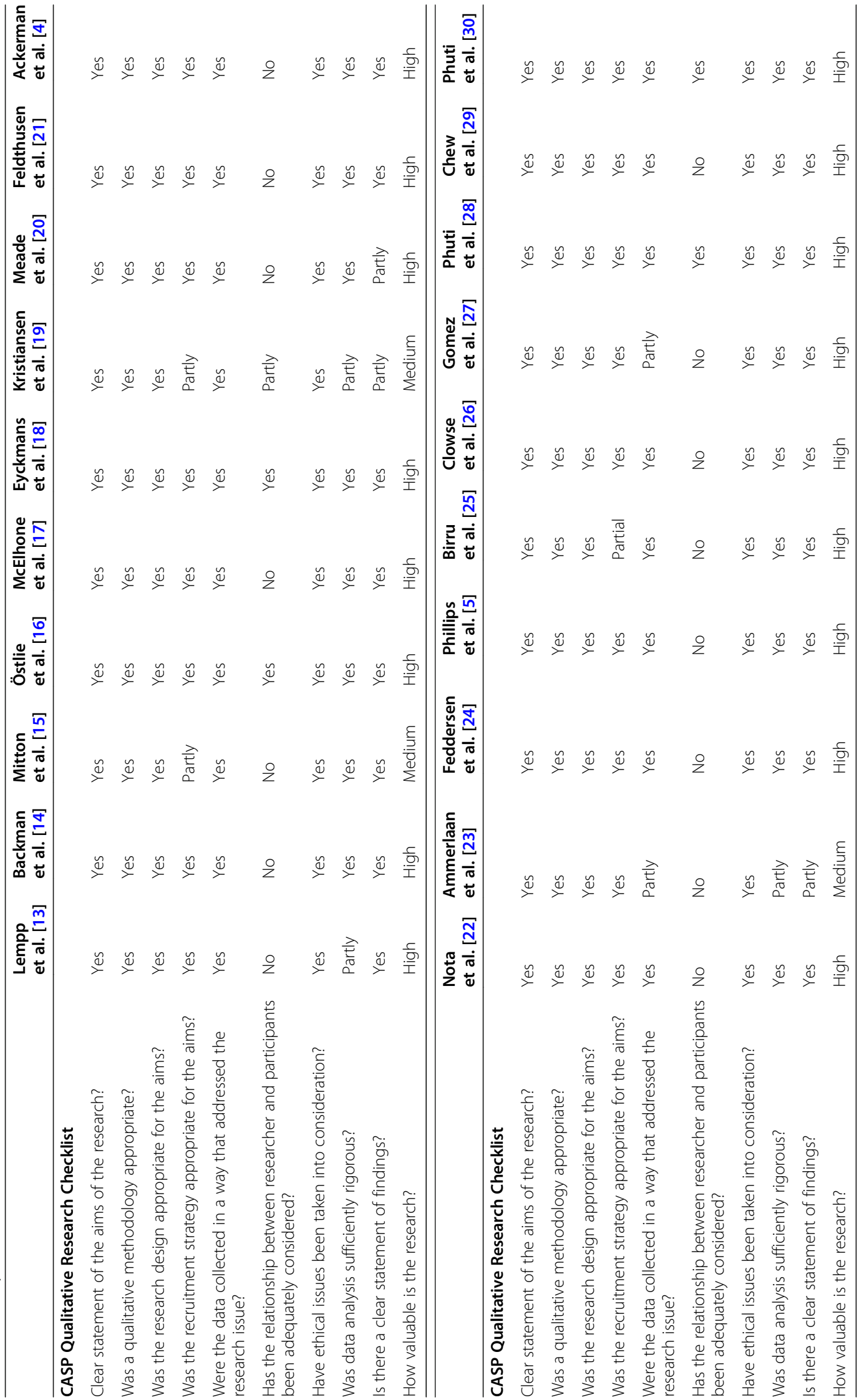


Table 3 Topics covered and illustrative text excerpts for descriptive themes

\begin{tabular}{|c|c|c|}
\hline Analytic theme & Descriptive theme & Topics covered \\
\hline \multicolumn{3}{|l|}{ Patient perspectives } \\
\hline $\begin{array}{l}\text { Making decisions } \\
\text { about planning } \\
\text { and experiencing } \\
\text { pregnancy }\end{array}$ & $\begin{array}{l}\text { Making decisions about } \\
\text { becoming pregnant }\end{array}$ & $\begin{array}{l}\text { - Factors impacting pregnancy decisions } \\
\text { - Fear of IA impeding ability to raise a child } \\
\text { - Childbearing alternatives (e.g., adoption) } \\
\text { - Pregnancy prevention and termination }\end{array}$ \\
\hline
\end{tabular}
$\begin{array}{ll}\text { Making decisions about } & \text { - Factors impacting medication decisions } \\ \text { using medications } & \text { - Wanting more information and decision }\end{array}$
Making decisions about - Factors impacting breastfeeding decisions breastfeeding - Wanting more information and decisional

Needing to prepare for pregnancy and parenthood support about breastfeeding
- Factors influencing conception and pregnancy planning

- Parenting while managing post-partum flares - Establishing support systems (e.g., family members, mothers with IA)
[Women] were careful in considering their capacity to manage pregnancies and motherhood. These considerations were important in their decisionmaking, and based on, as one participant commented: '....an honest appreciation of the reality of the situation' which is unlike that of 'other couples [who] get pregnant and then consider the consequences'.

One woman, who expressed fear of not being able to take care of a child and therefore had chosen not to have children, confronted another participant in the group.

'You had the courage to have children ... despite this (fatigue). I know that it is not an option for me ... where would I get the energy for that from?'

Medication safety was an important topic, and members expressed concerns over the use of biologics and prednisone during pregnancy, indicating that they would rather not take medications during the pregnancy if there were even a slight risk to the foetus.

'But nobody's told me what the side effects of steroids are during pregnancy, they just say it's kind of the safest option really and that they'll judge it when they get to it depending on how bad I am. So I'm walking into the unknown, I have no idea.'

'By about 4 weeks my mum was saying please stop breastfeeding and she'd been very probreastfeeding, but she could obviously see I was struggling (with mobility and pain), but I marched on and then at 6 weeks I dropped the child.'

The process of acceptance necessitated planning, as spontaneity regarding conception is not an option for women with RA. For some, it meant facing realities such as financial burdens: '...it is really important ... to have significant savings in the bank prior to falling pregnant. I did, which helped immensely because I was unable to continue the biologic medication, I was unable to work and had to rely on my savings to pay for medicine, doctors, etc.'

[One participant], who had been pregnant once, described openness in her pregnancy timeline because of her Lupus diagnosis: 'Yeah, so if I'm like 26 , and they are like, yeah, you are in remission, and I'm in a good place with everything else, I figure that might be the time. So I would not want to wait necessarily in case I get sick again.' Although [she] would prefer to wait until age 27 or 28 to become pregnant, she also recognized the need for flexibility in those plans because she could not predict the status of her lupus [...] Because of this, she said, 'Family planning for me would probably go around when I'm in remission.'

Prioritizing the needs of · Trade-offs between pregnancy/breastfeeding Another member described why, even after mother and child desires and potential harms of IA management - Self-advocacy

discussing the risks of pregnancy and the appropriate medications to take, women may move forward with their own pregnancy plan even against their rheumatologist's recommendation, 'I know [getting pregnant] is not the best for me...but I want to get pregnant.

'Two years, no child. Some of them [in-laws] are going to say, "You must take this one out, this is a barren [woman]."'

'With the rheumatologist advising certain
- Challenges with fertility, disease activity, and
conception

- Emotional impact of conception with IA
Experiencing parenthood 
Table 3 Topics covered and illustrative text excerpts for descriptive themes (Continued)

Analytic theme Descriptive theme Topics covered

Experiencing the impacts of disease on ability to be a mother

Making adjustments

Needing support

Worrying about

Navigating pregnancy and parenting with chronic disease unknown effects of

disease on pregnancy and parenting
Experiencing disease activity during pregnancy \& postpartum
- Perinatal flares and pregnancy complications

- Perinatal medication use
- Impacts of IA on motherhood (e.g., caring for children, breastfeeding)

- Impacts of IA on children

- Parenting strategies

- Childcare support

- Emotional and peer support

- Uncertainty of pregnancy progression, perinatal disease management, and ability to meet parental responsibilities

\section{Example quotations}

Ref.

medication-free timeframes before conception and the obstetrician/gynaecologist recommending surest ways to conceive on the first try, I felt like a science experiment for a while.'

It was the last four weeks of my pregnancy that I finally had some relief from RA but within a week of giving birth again I was in severe pain and not being able to lift my baby'.

[One participant] said she felt very depressed after giving birth, and it was hard to know if it was a post-partum flare in her RA or post-natal depression: Well they could not pinpoint it really, whether it was the flare in my arthritis or post-natal depression, all I did was cry, I felt like I was going mad.'

Two patients reported that the disease had limited their family size, in one case preventing her from having any children.

'You get to learn to do things differently as a parent with a chronic illness. My kids know I cannot pick them up too often, so they climb up onto me for cuddles. Some days we had PJ days and they loved it as much as I did'.

'I would not have been able to make this decision if I did not have the support from family and my husband'.

I'm just so nervous about taking anything it's like my gut instinct telling me not to. If I do and something goes wrong then I'd never forgive myself you know. At the same time I do not want to end up in so much pain that I cannot care for my baby. It's so scary.'

I'm hoping and praying that none of the [my] children will get this $[R A]$... because I would hate for any of them [my kids] to get arthritis. Well, you do not want them to be ill anyway, but you know, it's a condition that's not very kind.

Informants who were mothers were concerned about shortcomings in care and participation in their children's life, and about the children's health and chances of inheritance of rheumatic diseases.

Struggling to cope with • Coping with personal limitations (e.g., ability to For many women, their [autoimmune rheumatic personal limitations meet reproductive goals) diseases] led to them feeling restrained by their physical symptoms, and they felt that they were unable to do some of the things that 'normal' mothers do.

The sense [feeling] of not feeling entitled to something; it's painful. It's a bit emotional. If I cannot wash my clothes, if I cannot clean for myself or cannot carry heavy things, how is it going to be possible for me to maintain kids, you know?

II find it hard to come to terms with that. My mantra has always been, ever since I could speak as it were, that I wanted to have children. But I never had the nerve to take the initiative ... There are too many obstacles and limitations, I reckon, which stop me from ever having children. Those fears run deep. I would dearly love to have children, but I do not have the courage.

The women perform self-stigmatization in the form of self-blame and guilt when they cannot accomplish what they want in the role of mother because of fatigue, pain, or physical limitations. One of the women who had just given birth was disappointed and sorry that she did not live up to the expectations she had of herself as a future mother. $\begin{array}{ll}\text { Facing judgement from } & \text { - Navigating guilt and stigma } \\ \text { self and others } & \cdot \text { Encountering judgement from healthcare }\end{array}$ professionals and peers 
Table 3 Topics covered and illustrative text excerpts for descriptive themes (Continued)

\begin{tabular}{lll}
\hline Analytic theme & Descriptive theme & Topics covered \\
\hline $\begin{array}{l}\text { Finding value in } \\
\text { motherhood }\end{array}$ & - Source of purpose and motivation \\
& $\begin{array}{l}\text { Creating a self-identity } \\
\text { beyond their disease }\end{array}$ & $\begin{array}{c}\text { - Impact of self-identity (e.g., as a parent, care- } \\
\text { giver, employee) on reproductive decisions }\end{array}$
\end{tabular}

'My son and the baby that grows within me are my [20] shining light. They are the little beings that get me out of bed in the morning when I am so stiff and sore and riddled with pain that I do not know if I

can face the day'.

$\begin{array}{ll}\text { Creating a self-identity } & - \text { Impact of self-identity (e.g., as a parent, care } \\ \text { beyond their disease } & \text { giver, employee) on reproductive decisions }\end{array}$

Encountering poor understanding of disease

information and resources for pregnancy planning
- Feeling uninformed about pregnancy and IA management

- Encountering poor understanding from family, community members, and public agencies

The women prioritize and direct the bulk of their time and energy to their working life. This is interpreted as, first and foremost, their desire to identify with the role associated with working life, rather than the identity that is solely associated with motherhood or with [rheumatoid arthritis]. The women feel that the role of worker is attractive because it gives status, and because it allows them to be self-supporting.

The impact on women's identity was often discussed, with women wanting to be a 'normal' parent and to be seen as a whole person not a disease.

I'd say the first five years of having [rheumatoid arthritis] ... I had no idea, information was scarce, I

Seeking high quality, timely, consistent, and accessible information
- Patient-specific pregnancy information (e.g. disease heredity, fertility, pregnancy complications, perinatal disease management, birth choices, and breastfeeding)

- Information for early parenthood (e.g., practica strategies for managing pain/fatigue)

- Information addressing prevailing misconceptions about IA
Seeking support services
- Avenues for emotional support (e.g. counseling; peer support groups)

- Role of practical support services (e.g., social workers, hired help)

- Barriers to accessing support

Learning from peers - Pragmatic information and resources with similar experiences $\cdot$ Source of emotional support and motivation - Role of online forums/support groups had done millions of Google searches and went to libraries and there was nothing. Nothing about pregnancy, breastfeeding and [rheumatoid arthritis]. And even my doctors were really... did not seem to know much about what medications were safe, and they'd have to go and ring other doctors, and it was really just like... I thought gee, am I the first person in the entire world to have RA and be pregnant?'

'Some practical information as to how to do certain daily tasks without the use of certain limbs. So, you know, if my hands are so swollen that I cannot dress myself, how do I pick up my baby using my forearms, how do I... how can I carry my baby to minimise the impact on my arms and shoulders and back and wrists etc. Clothing to put the baby in... that was a big thing for me, you know, most baby clothes have... stupid press-studs, yeah, which were just the bane of my life back then. So and I managed to find clothing that was much easier for me to get on and off the baby - so that was, you know, that was a big thing. And, you know, even just.. every day household tasks, you know, tips as to how to, you know, reduce your fatigue and cut down on your household duties and to... minimise your energy output'

'I think my problems differ from older people. I was just looking for people who work, for people with young children and how they manage their lives while having a rheumatic disease. I also wonder how I could combine work and home.'

Some commented on the lack of resources for mothers with arthritis and desired a person or place to go to when facing specific challenges. Participants referred to 3 types of support: practical (help with child care or cleaning), emotional (help dealing with feelings), and moral (someone to listen and share). Sources of support included family and friends, health professionals, and hired help.

Members stated that participating in the online community was a positive way for them to share concern, anxiety, or questions they had about arthritis and pregnancy.

Discussing with others how best to talk to your children about your rheumatic disease

[...] Hearing from others how they involve their partner in child care

[...] Hearing from fellow patients how they decided whether or not to have children 
Table 3 Topics covered and illustrative text excerpts for descriptive themes (Continued)

\begin{tabular}{|c|c|c|}
\hline Analytic theme & Descriptive theme & Topics covered \\
\hline $\begin{array}{l}\text { Interacting with } \\
\text { healthcare } \\
\text { providers }\end{array}$ & $\begin{array}{l}\text { Receiving care from } \\
\text { several providers }\end{array}$ & $\begin{array}{l}\text { - Value of multi-disciplinary healthcare } \\
\text { - Barriers to patient satisfaction (e.g., care } \\
\text { coordination/continuity, provider knowledge) }\end{array}$ \\
\hline
\end{tabular}

Women's experiences varied widely, but most felt

Seeking and receiving information

Requiring compassionate and
- Information on medication use and safety preconception and during pregnancy

- Role of trust in patient-provider relationship

- Approaches to compassionate patient communication

- Value of provider knowledge about IA and reproductive care

\section{Provider perspectives}

Providing reproductive healthcare
Understanding professional responsibility
Discussing pregnancy $\quad$ Value of timely, collaborative discussions planning
- Barriers to discussing reproductive goals (e.g. patient-provider relationship) holistic care

that there was a lack of well-coordinated multi-

disciplinary management between different secondary care departments, as well as primary care, and this could undermine women's trust.

I've always been the go between, the departments do not really talk to each other and I've many a time been in a position when I, I've said to either my GP [general practitioner] or my consultant you have lied to us because you are both telling me different things.'

It's confusing - the doctors from haematology told me that chloroquine is not safe for the pregnancy, must stop it. At rheumatology the doctors said it's OK for the baby. At haematology they told me that must stop breastfeeding her because I must go back to the medication.'

Most women, regardless of education level or socioeconomic status, reported feeling intimidated by their rheumatologist and therefore hesitant to speak up about their true pregnancy desires.

Younger women described feeling that they needed to get the doctor's permission if they wanted to embark on a pregnancy.

Focus group participants also expressed trust in their rheumatologist, but regularly sought additional sources of information (primarily from online sources) in order to make informed treatment decisions.

Good communication, clear advice, being open to questions, compassion, kindness, understanding, encouragement, and honesty from health professionals were viewed as being important aspects of care.

All rheumatologists expressed a sense of responsibility to provide some aspects of [family planning counseling and reproductive health care] to female patients of reproductive age. When asked how they defined [family planning counseling and reproductive health care], rheumatologists' definitions unanimously centred on clarifying women's pregnancy intentions and timing, educating patients about the associations between their diseases and pregnancy, and optimizing women's health and anti-rheumatic drug regimens in anticipation of pregnancy.

There is a tendency to say, "well, somebody else will talk to [patients] about contraception, somebody else will talk to them about family planning".

Desiring more guidance $\cdot$ Challenges of clinical decision-making and patient counseling for pregnancy

- Need for increased knowledge about IA, pregnancy, and motherhood among healthcare providers

Rheumatologists consistently expressed that they wanted access to consensus guidelines that gave them clear recommendations for managing diseases and anti-rheumatic drugs for women before, during, after pregnancy, and through lactation.

We suspect that these knowledge gaps and the rheumatologists' lack of confidence in drug safety make it difficult for them to discuss medication use in pregnancy with their patients in a way that would encourage a woman to take the medication.

Fearing negative $\quad$ - Impact of rheumatologists' fear of managing outcomes high-risk pregnancies on patient-provide relationship

Most rheumatologists could recall at least one pregnancy conceived on a teratogenic medication or during a period of high disease activity. Some were afraid of managing this complicated situation and having little control of the outcome: "That's part of the fear, I think ... They come in crashing, and 
Table 3 Topics covered and illustrative text excerpts for descriptive themes (Continued)

\begin{tabular}{lll}
\hline Analytic theme & Descriptive theme & Topics covered \\
\hline Having time restrictions & $\begin{array}{c}\cdot \text { Impact of time restrictions on reproductive } \\
\text { care provision }\end{array}$
\end{tabular}

Interacting with patients

Discussing pregnancy planning
Providing counseling and managing medication use

Building the patientprovider relationships

- Challenges building patient-provider relationships

- Role of patient trust in adherence to medical advice

- Approaches to reproductive care and perinatal medication use counseling

- Challenges managing IA pregnancies and providing medication information

discussions

pregnancy intentions

- Factors facilitating pregnancy planning discussions

\section{Example quotations}

Ref

then it's like you are responsible, and you are not sure that you are going to be able to fix that.'

Most rheumatologists expressed that competing priorities during clinic visits limited their ability to provide [family planning counseling and reproductive health care]. As one rheumatologis stated, '[There is] pressure to see patients in the shortest amount of time... I focus on things that only I as a rheumatologist could focus on - the disease process... there is a tendency to say, "Well, somebody else will talk to [patients] about contraception, somebody else will talk to them about family planning."'

As one rheumatologist stated, When you asked me about how many pregnant patients that I have had, despite the fact that I feel pretty comfortable discussing contraception, my heart did skip a beat And I thought, you know, it's not one of the most pleasant things to deal with in my practice. And it's because of the fear. There is a fear that, what if something goes wrong?... I think that we are all always concerned that anything could happen, something could go wrong...

Another rheumatologist noted the importance of bringing up the topic of pregnancy to aide in pregnancy planning, saying, 'If you do not bring it up, they'll just get pregnant.'

Some rheumatologists use a different approach to discuss medication use in pregnancy that appealed to the patient's desire for a healthy baby. These rheumatologists discussed medication use in pregnancy as a benefit to the baby, not primarily the mother:

'I think they care more about the baby's health than their own, so if I show them that there's data that the baby actually has better outcomes in pregnancy if they are on the [medication], then they are more willing to do it. But if you say, "You're gonna do better," then they are not as convinced. So you have to convince them that it's better for the baby.'

Another rheumatologist described how feelings of fear and anxiety fractured the relationship with a newly pregnant patient, 'I think I wasn't able to build up a very good physician-patient relationship, because I think that I got so scared that I kind of blurted out all the data for every single one of the drugs that we were talking about. I think that kind of scared her [patient]. She did not follow up very well... the pregnancy went well, but she never really did the follow-up as well as she should, and I felt it was because she wasn't trusting my judgment.'

One rheumatologist described the patient dilemma when receiving care for their disease during pregnancy as, 'does this person want to hurt me and my baby, or do they want to help me and the baby?'

As one rheumatologist described: 'Rheumatology and OB-GYN [obstetrics-gynaecology], it should be a collaboration. I do not think it's reasonable to expect family practitioners [to manage reproductive care] when they manage so many diseases and so many medications.'

'It's actually getting everybody that might need to be involved to see it in a more holistic way.'

Health professionals also felt that it was important to consider the specific support needs of women
Providing support services - Role of tailored support services for IA (e.g.,
pre-conception counseling, social/

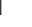

Coordinating patient care with
other providers $\begin{array}{ll}\text { Collaborating on } & \text { - Value of multi-disciplinary collaboration } \\ \text { patient care planning } & \text { - Barriers to patient care coordination }\end{array}$ 
Table 3 Topics covered and illustrative text excerpts for descriptive themes (Continued)

\begin{tabular}{|c|c|c|c|c|}
\hline Analytic theme & Descriptive theme & Topics covered & Example quotations & Ref. \\
\hline & & psychological supports) & $\begin{array}{l}\text { with [autoimmune rheumatic diseases]. } \\
\text { 'I'm just thinking about the, there's very specific } \\
\text { anxiety and concerns that come for these women in } \\
\text { the context of their parenting role having a chronic } \\
\text { sort of autoimmune disorder.' }\end{array}$ & \\
\hline
\end{tabular}

consequences of changing or discontinuing medications on disease activity and subsequent medication efficacy $[4,5,20,29]$, alternative or adjunct (e.g. folic acid supplementation) therapies [4, 29], and lifestyle modifications $[4,29]$. Factors influencing medication decisions included concerns about medication impacts on foetal development [29] and medication discontinuation on disease management and pregnancy $[20,27]$ as well as desiring to experience pregnancy [26] and breastfeeding $[5,20]$. Sources of medication information comprised healthcare providers, including rheumatologists, nurses, and pharmacists $[4,26,27,29]$, as well as online sources $[26,29]$. Patients indicated desiring more decisional support related to medication use, particularly guidance from providers $[4,5]$.

Patients recounted needing to prepare for pregnancy and parenthood, including timing conception, making medication adjustments, achieving low disease activity, and recognizing potential complications (e.g., miscarriage) $[5,16,20,27,29,30]$. Patients also identified needing to prepare for parenting while managing post-partum flares and establishing support systems that include family members and mothers with similar experiences [5, 20, 29].

Many patients described having to balance prioritizing the needs of mother and child [4, 20, 24, 26, 28-30]. Patients shared grappling with trade-offs between their desires to become pregnant and breastfeed and to avoid potential harms from medication use, disease flares, and permanent joint damage $[4,26,29]$. They reported selfadvocacy was instrumental to realizing their reproductive goals [4, 28-30].

\section{Experiencing pregnancy and parenthood}

Patients' experiences of pregnancy and early parenthood were intertwined with their disease management, expectation of pregnancy and parenting, and ability to access support, which was captured by 5 descriptive themes in 16 studies [4, 5, 13-15, 17-21, 24, 26-30].

Patients reported challenges when trying to conceive related to fertility, disease activity, and pressure to conceive quickly after discontinuing certain medications $[13,15,18-20,28,30]$. These were noted to be sources of stress, fear, and emotional hardship [18, 20, 28, 30]. Some patients felt they needed permission from their provider to conceive $[19,28]$. Many reported experiencing disease activity during pregnancy and post-partum, describing worsening symptoms, limitations preforming daily tasks, requiring support from caregivers, and experiencing pregnancy complications and hospitalization $[4,13-15,20$, $27,29,30]$. For some, these hardships impeded their ability to work and posed financial challenges [20, 29]. While some patients resolved not to take medications perinatally despite experiencing disease-related challenges [14, 29], others described needing to use medications due to disease severity or worsening symptoms $[4,20,29]$. Some patients experienced disease remission during pregnancy $[14,20$, 29]. Several patients who restarted medications postpartum found they were no longer effective and had to seek new therapies [20].

Patients described experiencing the impacts of disease on ability to be a mother, which included navigating pain and fatigue, difficulty physically caring for children, and being unable to breastfeed due to physical limitations or medications used for post-partum flares $[5,14,15,17$, 20, 21, 24, 28, 29]. Patients reported making adjustments to accommodate for personal disability $[5,13-15,20,24$, 29]. Parenting strategies included choosing to stay at home or arranging flexible work hours, prioritizing rest and self-care, planning family activities requiring limited physical activity, and teaching children to self-sooth [14, $20,24,29]$. Some patients reported pride in raising highly independent children, although this was also a source of guilt $[14,20]$. Ultimately, patients reported needing support for the practical and physical demands of pregnancy and parenthood [5, 13, 14, 17, 20, 21, 26, 29]. Many depended on support enlisted from extended family, while others received limited assistance [5, 13, 14, 17, 20, 21, 29]. Additionally, patients valued emotional and peer support from online communities [5, 26, 29].

\section{Navigating caregiving with chronic disease}

Reproductive uncertainty related to IA placed a heavy burden on patients' emotional and psychological wellbeing. We identified 5 descriptive themes about the impacts of being a caregiver with a chronic debilitating disease in 17 studies $[4,5,13-20,23,24,26-30]$.

Many patients reported worrying about unknown effects of disease on pregnancy and parenting $[4,5,13,15-18,20$, 23, 24, 27-30]. Patients struggled with uncertainty related to managing their disease activity during pregnancy and 


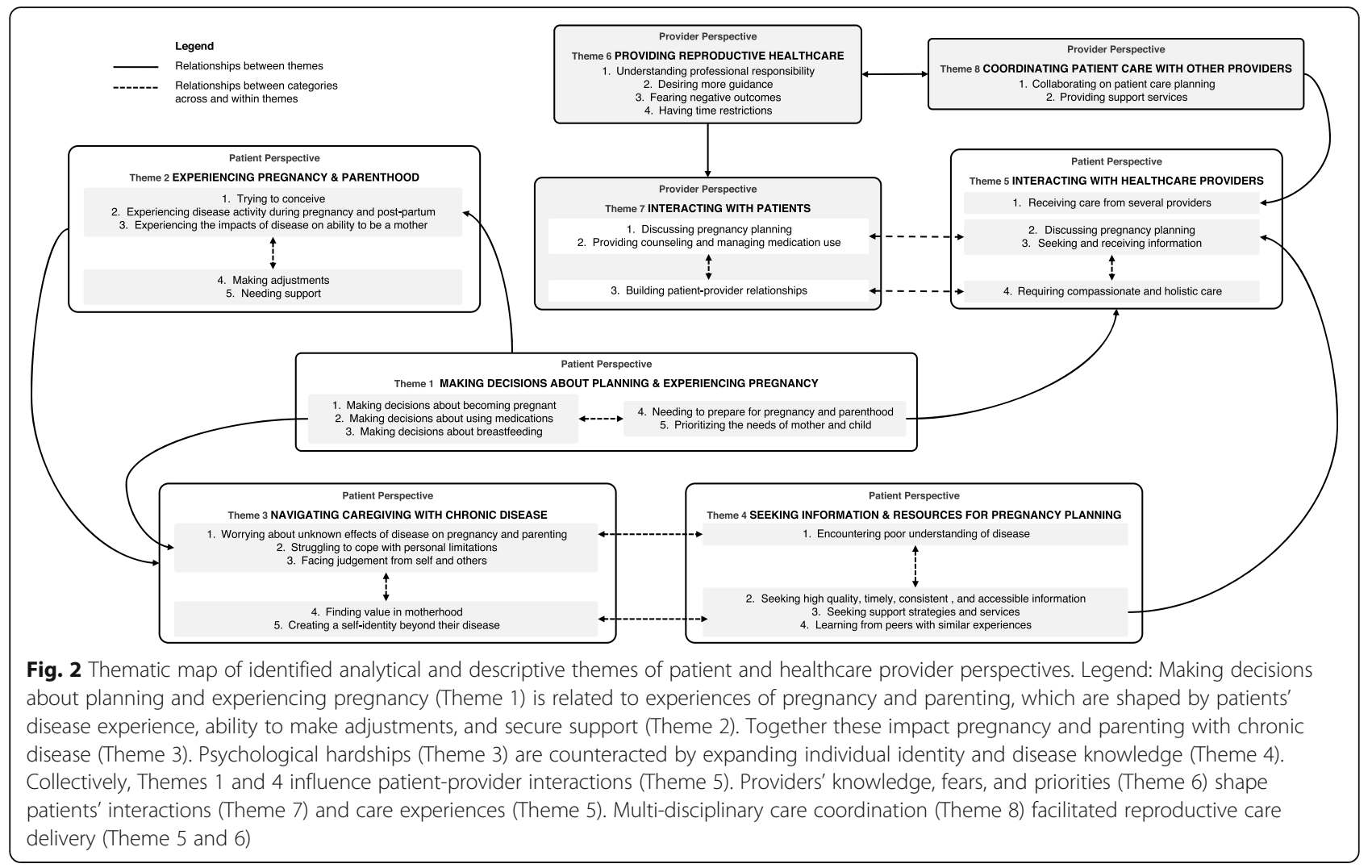

post-partum $[4,15,16,20,28-30]$. They shared concerns about fertility $[5,18,28,30]$, timing conception $[5,20]$, passing their disease to their children $[5,13,16-18,23$, $30]$, experiencing pregnancy complications $[5,17,18,27$, $28,30]$ or disease progression $[5,18,20,24]$, causing foetal harm with medication use [18, 20, 29], and being unable to meet their parental responsibilities (e.g., taking care of children, financially providing for family) $[5,16,18-20$, 29]. Accordingly, patients described the emotional impacts of struggling to cope with personal limitations related to their disease $[4,5,15,19,20,24,28-30]$. Patients felt isolated, anxious, frustrated, beaten down, and useless $[4,5,15,18,20,28-30]$ as well as restricted in their ability to meet their reproductive goals $[18,30]$.

Patients described facing judgement from self and others $[4,14-16,20,24,26,28,30]$. They associated stigma with their disease, describing IA as an invisible disability' [24]. They recounted feeling guilty for requiring help at home and accommodations at work, as well as their children having to develop independence at an early age $[15,16,20,24]$. Patients shared experiencing judgement and lack of understanding from healthcare professionals regarding their pregnancy intentions, medication decisions, and uptake of medical advice $[4,14,26]$ as well as members of their community regarding their ability to become pregnant and fulfil their parental responsibilities $[15,28,30]$.
Despite challenges, patients described finding value in motherhood as a driving force for pursing pregnancy and their children as a source of purpose and motivation $[5,15,20,24,28,29]$. Additionally, they depicted creating a self-identity beyond their disease as a parent, caregiver, and employee [5, 13, 15, 20, 24]. Patients who had children wanted to be seen as 'normal' parents $[5,15]$, while those who perceived parenthood was not in their best interest found other goals to pursue [20].

\section{Seeking information and resources for pregnancy planning} Regarding knowledge gaps concerning pregnancy planning with IA, we identified 4 descriptive themes related to seeking information about pregnancy planning and disease management in 11 studies $[4,5,13-15,20,23$, 26, 28-30].

Patients reported encountering poor understanding of disease personally and from their family, community members, and public agencies providing support resources $[4,14,20,28,30]$. Patients voiced feeling uninformed and receiving limited information about pregnancy and disease management from healthcare providers $[4,5,15,20,30]$. Overwhelmingly, patients shared challenges when seeking high quality, timely, consistent, and accessible information about perinatal disease management $[4,5,15,23,26,29$, 30], emphasizing the importance of specific and tailored information given maternal stage and health literacy $[4,5,23$, 
29]. Prior to pregnancy, patients wanted to receive information about disease heredity, fertility, pregnancy planning, pregnancy complications, perinatal disease management (e.g., medication use and discontinuation), birth choices (e.g., natural birth, caesarean), and breastfeeding $[4,5,15$, $23,29]$. Additionally, patients emphasized wanting information and support for early parenting related to pain and mobility, including practical strategies for adjusting to daily challenges $[4,5,23]$. Most reported seeking written information that can be accessed electronically [4, 15, 26, 29]. They also identified needing information addressing prevailing misconceptions about IA to be available for family, friends, employers, and public agencies [4, 5, 30].

Relatedly, patients described seeking support strategies and services [4, 5, 13, 14, 23, 29]. Patients emphasized wanting more avenues for emotional support, including counseling and peer support groups, for addressing feelings of uncertainty and isolation $[4,5,14,29]$. Patients depicted the role of practical support services, such as social workers, nurses, and hired help, for assisting in household tasks, childcare, and activities of daily living [4, 5, 13, 14, 23]. Patients also described seeking practical strategies for minimizing pain and fatigue when caring for a baby (e.g., bathing, dressing, and feeding tasks) and information about assistive devices [4, 5, 14]. Barriers to accessing support were regional variability of available services, travel requirements for specialized services, existing services not accounting for the needs of patients with IA (e.g., perinatal classes that are physically strenuous), and receiving continuity of care [5].

Finally, patients expressed valuing learning from peers with similar experiences through shared personal concerns, experiences, and advice [4, 5, 23, 26, 29]. Patients were interested in pragmatic information and resources used by peers and further identified peers as sources of emotional support and motivation [4, 23, 29]. The majority of patients participated in online forums or support groups [5, 26, 29].

\section{Interacting with healthcare providers}

We identified 4 descriptive themes in 9 studies related to patients' experiences interacting with healthcare providers [4, 5, 14, 15, 20, 26, 28-30].

Patients discussed their experiences receiving care from several providers, including rheumatologists, primary care physicians, obstetricians, counselors, physiotherapists, occupational therapists, midwives, and pharmacists $[4,5,20,29,30]$. They valued receiving care from a multi-disciplinary team when possible $[5,29]$. Patients who reported dissatisfaction with their care team mentioned issues of inadequate care coordination, a lack of care continuity, and a high frequency of appointments [5]. Some described receiving advice and encountering judgement from allied health professionals with poor disease understanding $[4,5,14,30]$.

Patients also shared diverse experiences discussing pregnancy planning with their providers [4, 5, 14, 15, 20, $26,29]$. They emphasised desiring timely and collaborative conversations about pregnancy planning $[5,15,26]$. Patients who reported positive patient-provider relationships shared establishing a plan for when they decide to start trying to conceive [29]. Those who reported concerns discussing their reproductive goals with providers shared feeling intimidated, fearing judgement, wanting to avoid confrontation, and not receiving support for becoming pregnant $[5,14,15,26,29]$.

Nevertheless, patients turned to their healthcare providers when seeking and receiving information about pregnancy planning $[4,5,15,26,29,30]$. Information from providers predominantly pertained to medication use and safety pre-conception and during pregnancy [29]. Rheumatologists were identified as the primary source of medical information, although some patients also obtained information from online sources, including forums and social media [4]. Trust in their provider influenced whether patients decided to follow providers' recommendations or seek information from other sources [4, 26]. Factors influencing provider trust included the amount of information shared about pregnancy with IA, the consistency of information received from their healthcare team, their information preferences, and the provider's confidence as perceived by the patient $[4,5,30]$.

Overwhelmingly, patients indicated requiring compassionate and holistic care from providers [5, 14, 15, 28, 29]. Patients valued providers who were educated in their disease, particularly relating to reproductive care, supported their health and reproductive goals, and provided proactive care based on their individual patient needs beyond medication use [5, 14, 15, 28, 29].

\section{Provider perspective \\ Providing reproductive health care}

Reproductive care provision was influenced by the provider's perceived professional responsibility for meeting their patients' reproductive goals, their fears of responsibility for negative outcomes, and their capacity to incorporate reproductive care into their practice. We identified 4 descriptive themes related to the provider's perspective of delivering reproductive care in 4 studies $[4,5,25,26]$.

Providers, primarily rheumatologists, discussed understanding their professional responsibility [5, 25]. Overall, rheumatologists believed providing some reproductive care was within their professional responsibility, including clarifying pregnancy intentions, educating patients about pregnancy with IA, and optimizing patient's medication and disease management in anticipation of pregnancy 
[25]. This definition did not include contraception or abortion care [25]. While rheumatologists recognized it was their responsibility to ensure reproductive age patients on teratogenic medications were using contraception, they focused on managing their disease [25]. They preferred that primary care physicians or gynaecologists prescribe contraceptives [25]. Generally, providers agreed pre-conception counseling was necessary for patients with IA; however, they were unclear about which profession should provide this service [5].

Providers emphasised desiring more guidance for providing reproductive care $[5,25,26]$. Rheumatologists reported that clinical decision-making and patient counseling was challenging given gaps in clinician knowledge about medication safety in pregnancy [26]. They consistently expressed wanting consensus guidelines with clear recommendations for managing diseases and medication use for patients before, during, and after pregnancy and for breastfeeding [25]. Both providers and patients recognized the need for increased awareness of IA and its impact on pregnancy and motherhood among healthcare providers caring for IA patients [5].

Notably, rheumatologists described the impacts of fearing negative outcomes on their patients [25, 26]. Most rheumatologists described experiencing tension between respecting patients' autonomy to become pregnant and fear of managing high-risk pregnancies and having limited control of pregnancy outcomes [25, 26]. Many had difficulty understanding why patients chose to pursue pregnancy given their risk of complications [26]. Some rheumatologists recognized their own fear may influence how they counsel patients [25]. Additionally, having time restrictions was reported as a barrier to providing reproductive care $[4,25,26]$.

\section{Interacting with patients}

Providers' experiences interacting with patients were influenced by their abilities to hold space for patient's desires amidst their personal beliefs, to harness trust, and to engage patients in discussions about reproductive health, which was captured by 3 descriptive themes in 3 studies $[5,25,26]$.

Providers shared their experiences discussing pregnancy planning with their patients [5, 25, 26]. Some rheumatologists recognized they may overestimate the magnitude of pregnancy risks and that their caution may discourage patients from discussing their reproductive goals and place undue pressure on patients to avoid pregnancy [25]. As pregnancy needs to be planned carefully with IA, rheumatologists acknowledged the importance of being aware of patients' pregnancy intentions and usually respected their patients' autonomy to pursue pregnancy $[5,25,26]$. Factors facilitating pregnancy planning discussions included patient initiation, reproductive decisiveness, and having a female provider [25, 26].

Providers also discussed providing counseling and managing medication use $[5,25,26]$. Rheumatologists felt most comfortable discussing medications for perinatal disease management [25]. Some recommend pre-natal folic acid supplementation; however, most felt uncomfortable prescribing contraceptives [25]. Providers described medically ill-timed pregnancies (i.e., conception while taking a teratogenic medication or experiencing high disease activity) and medication non-adherence during pregnancy as dilemmas in care [26]. Noted challenges of providing medication information were communicating medication risk to patients, knowledge gaps about medication safety in pregnancy, and confidence in available data $[25,26]$. Given the recognized desire to avoid foetal harm among patients, some rheumatologists noted the value of framing discussions about medication use as a benefit to both mother and baby [26]. Overall, providers underlined needing to offer patients pre-conception counseling and timely high quality written information for pre-conception, pregnancy, and post-partum [5].

Finally, providers discussed challenges of building patient-provider relationships $[5,25,26]$. Several rheumatologists acknowledged that a 'gloom and doom' approach towards pregnancy planning diminished patient trust $[25,26]$. Trust was recognized to contribute to patient adherence to medical advice [26]. Distrust of the medical community was noted to persist in marginalized communities [26].

\section{Coordinating patient care with other providers}

We identified 2 descriptive themes related to coordinating patient care in 3 studies $[5,25,26]$.

Providers, like patients, recognized the value of collaborating on patient care planning with a multi-disciplinary team, including obstetrician-gynaecologists, nurses, midwives, and occupational therapists [5, 25, 26]. Key barriers to patient care coordination included limited communication between providers and regional infrastructure for specialized multi-disciplinary teams, leading to unnecessary lab testing and inconsistent medication recommendations $[5,26]$.

Moreover, providers recognized the need for providing support services and tailored care to IA patients [5, 25]. Identified services included pre-conception counseling, social and psychological supports for the emotional impacts of high-risk pregnancy and parenting with IA, and social support for the practical challenges of pregnancy and early parenting $[5,25]$.

\section{Discussion}

We thematically synthesized 20 qualitative studies on the pregnancy and early parenting experiences of 368 
patients with IA and 51 providers. Our conceptual model illuminates the complex relationships between patient and provider perspectives about reproductive care provision, patients' experiences of IA and pregnancy, and patients' processes for seeking information and making reproductive decisions. Our analysis suggests how informed patients felt, how well their IA was managed, and how capable they were of accessing support informed the acceptability of their pregnancy and early parenting experiences.

For providers, our results illuminate the necessity of collaborative, comprehensive, and patient-centred approaches to delivering reproductive care. There is room for improving how providers build trust, support patients' reproductive goals and expectations, and collaborate in making decisions, while in turn building their professional confidence in reproductive care provision to patients with IA $[5,14,15,25,26,28,29]$. Moreover, patients would benefit from comprehensive care beyond medication use for IA through multi-disciplinary care coordination of their healthcare team, connecting to peers with similar experiences, and receiving referrals to psychological and social services and resources $[4,5,14,25,26,29]$.

For patients, our review highlights measures within a patient's control with the potential to improve their reproductive care experience. It is important that patients have conversations with their providers about pregnancy planning early to reduce potential hardships related to achieving low disease activity preconception, timing pregnancy, making medication adjustments, and preparing for potential complications $[5,20,29]$. As such, patients must feel comfortable discussing pregnancy planning and their pregnancy intentions with providers. When seeking a referral for alternative or additional support, patients may consider asking potential providers about their knowledge and experience providing reproductive care to patients with IA. Beyond clinical disease management, patients may benefit from practical and emotional support services to mitigate the personal impacts of pregnancy and parenting with IA $[5,25]$.

It is important to contextualize our review with the release of perinatal guidelines for medication use in IA in 2016 and 2020 [31-34]. We included articles published from 2006 to 2020 . Studies specifically exploring pregnancy $[4,5,20,25,26,29,30]$, as opposed to parenting with IA, first appeared in 2012 with the majority published between 2018 and 2020 . This included few studies $(n=3)$ on the provider perspective $[5,25,26]$, which depicted the need for professional guidance for rheumatologists providing reproductive care, including recommendations for managing perinatal disease activity and medication use, despite the release of aforementioned guidelines.
Strengths and limitations of our review warrant discussion. We developed and employed a comprehensive search with research librarians to capture published studies reporting on pregnancy and early parenting among female patients with IA. We used established methods for evaluating reporting of qualitative studies and synthesizing qualitative results $[9,11,12]$, which provided transparency of our data analysis and interpretation processes. An inductive approach ensured themes were derived from the data and two authors reviewed the coding framework and thematic synthesis to enhance the credibility of findings. Some studies provided limited participant demographic information, which may affect the adaptability of our results. It is also possible that the identification of relevant studies was limited by publication bias; however, as qualitative synthesis aims to provide interpretive explanation rather than predictions, sampling is meant to be purposive as opposed to exhaustive [12, 35]. Finally, our analysis was limited by the scope of research in this area, which to-date has not extensively explored the intersectional influences of gender and race on patient's reproductive experiences or their experiences managing contraception and terminating pregnancies.

Our review provides a conceptional understanding of the reproductive care experiences of female patients with IA and their providers. It is critical for providers to recognize the toll of uncertainty felt by patients 'walking into the unknown' of navigating pregnancy and early parenthood. Enhancing the degree of support felt by individuals with IA by engaging in open conversations about pregnancy planning and shared decision-making may empower patients and strengthen patient-provider relationships. Interventions focused on facilitating access to reliable and timely information and practical and emotional support may help lessen the personal impacts of pregnancy planning and parenting with IA.

\section{Abbreviations}

AS: Ankylosing spondylitis; CASP: Critical Appraisal Skills Programme; ENTR

EQ: Enhancing transparency in reporting the synthesis of qualitative research; GP: General practitioner; IA: Inflammatory arthritis; JIA: Juvenile idiopathic arthritis; OB-GYN: Obstetrics-gynaecology; PsA: Psoriatic arthritis;

RA: Rheumatoid arthritis; SLE: Systematic lupus erythematosus

\section{Supplementary Information}

The online version contains supplementary material available at https://doi. org/10.1186/s13075-021-02493-z.

\section{Additional file 1.}

Acknowledgements

Not applicable.

Authors' contributions

All authors were involved in drafting the article or revising it critically for important intellectual content, and all authors approved the final version to be submitted for publication. MDV had full access to all of the data in the study and takes responsibility for the integrity of the data and the accuracy 
of the data analysis. Study conception and design: NR, UE, VK, and MDV. Acquisition of data: UE and VK. Analysis and interpretation of data: NR, RG, UE, VK, SM, GH, NA, NB, and MDV.

\section{Funding}

Dr. De Vera holds a Tier 2 Canada Research Chair and is a recipient of a Scholar Award from the Michael Smith Foundation for Health Research. Dr. Sarah Munro is a recipient of a Scholar Award from the Michael Smith Foundation for Health Research. Nevena Rebić is a receipt of a CIHR Drug Safety and Effectiveness Cross-Disciplinary Training (DSECT) programme award. This research was funded by a grant from Canadian Initiative for Outcomes in Rheumatology Care.

\section{Availability of data and materials}

Data sharing is not applicable to this article as no datasets were generated or analysed for the study.

\section{Declarations}

Ethics approval and consent to participate

Not applicable.

\section{Consent for publication}

Not applicable.

\section{Competing interests}

The authors declare that they have no competing interests.

\section{Author details}

${ }^{1}$ Faculty of Pharmaceutical Sciences, University of British Columbia, 2405 Wesbrook Mall, Vancouver, BC V6T 1Z3, Canada. ${ }^{2}$ Arthritis Research Centre of Canada, 5591 Number 3 Rd, Richmond, BC V6X 2C7, Canada. ${ }^{3}$ Collaboration for Outcomes Research and Evaluation, 2405 Wesbrook Mall, Vancouver, BC V6T 1Z3, Canada. ${ }^{4}$ University of British Columbia Library, 2198 Health Sciences Mall, Vancouver, BC V6T 1Z3, Canada. ${ }^{5}$ Department of Obstetrics and Gynaecology, University of British Columbia, Suite 930, 1125 Howe Street, Vancouver, BC V6Z 2K8, Canada. ${ }^{6}$ Centre for Health Evaluation and Outcome Sciences, 588-1081 Burrard Street St. Paul's Hospital, Vancouver, BC V6Z 1Y6, Canada. ${ }^{7}$ Cumming School of Medicine, University of Calgary, 3330 Hospital Drive NW, Calgary, Alberta T2N 4N1, Canada. ${ }^{8}$ Division of Rheumatology, Department of Medicine, Faculty of Medicine, University of British Columbia, 802-1200 Burrard Street, Vancouver, BC V6Z 2C7, Canada. ${ }^{9} \mathrm{School}$ of Populations and Public Health, University of British Columbia, 2206 East Mall, Vancouver, BC V6T 1Z3, Canada.

Received: 29 September 2020 Accepted: 29 March 2021 Published online: 21 April 2021

\section{References}

1. Badley EM, Kasman NM. The impact of arthritis on Canadian women. BMC Womens Health. 2004;4(1):S18.

2. Andreoli L, García-Fernández A, Chiara Gerardi M, Tincani A. The course of rheumatic diseases during pregnancy. Isr Med Assoc J. 2019;21(7):464-70.

3. Chakravarty E, Clowse MEB, Pushparajah DS, Mertens S, Gordon C. Family planning and pregnancy issues for women with systemic inflammatory diseases: patient and physician perspectives. BMJ Open. 2014;4(2):e004081.

4. Ackerman IN, Jordan JE, Van Doornum S, Ricardo M, Briggs AM. Understanding the information needs of women with rheumatoid arthritis concerning pregnancy, post-natal care and early parenting: a mixedmethods study. BMC Musculoskelet Disord. 2015;16(1):194.

5. Phillips R, Pell B, Grant A, et al. Identifying the unmet information and support needs of women with autoimmune rheumatic diseases during pregnancy planning, pregnancy and early parenting: mixed-methods study. BMC Rheumatol. 2018;2(1):21

6. Proulx L, Richards D, Robertson N, Wilhem L. Pregnancy and parenting with arthritis: bridging the information gap. Paper presented at: Canadian Rheumatology Association. 2015; Quebec, QC

7. Langen ES, Chakravarty EF, Liaquat M, El-Sayed YY, Druzin ML. High rate of preterm birth in pregnancies complicated by rheumatoid arthritis. Am J Perinatol. 2014;31(1):9-14
8. Tong A, Flemming K, Mclnnes E, Oliver S, Craig J. Enhancing transparency in reporting the synthesis of qualitative research: ENTREQ. BMC Med Res Methodol. 2012;12(1):181.

9. Critical Appraisal Skills Programme (CASP). CASP qualitative checklist https:// casp-uk.net/casp-tools-checklists/. Published 2020. Accessed 28 Sep 2020.

10. Majid U, Vanstone M. Appraising qualitative research for evidence syntheses: a compendium of quality appraisal tools. Qual Health Res. 2018;28(13):2115-31.

11. Thomas J, Harden A. Methods for the thematic synthesis of qualitative research in systematic reviews. BMC Med Res Methodol. 2008:8:45.

12. Barnett-Page $E$, Thomas J. Methods for the synthesis of qualitative research: a critical review. BMC Med Res Methodol. 2009;9:59.

13. Lempp H, Scott D, Kingsley G. The personal impact of rheumatoid arthritis on patients' identity: a qualitative study. Chronic IIIn. 2006;2(2):109-20.

14. Backman CL, Smith Ldel F, Smith S, Montie PL, Suto M. Experiences of mothers living with inflammatory arthritis. Arthritis Rheum. 2007:57(3):381-8.

15. Mitton DL, Treharne GJ, Hale ED, Williams RA, Kitas GD. The health and life experiences of mothers with rheumatoid arthritis: a phenomenological study. Musculoskeletal Care. 2007;5(4):191-205.

16. Ostlie IL, Johansson I, Möller A. Struggle and adjustment to an insecure everyday life and an unpredictable life course. Living with juvenile idiopathic arthritis from childhood to adult life - an interview study. Disabil Rehabil. 2009;31(8):666-74.

17. McElhone K, Abbott J, Gray J, Williams A, Teh LS. Patient perspective of systemic lupus erythematosus in relation to health-related quality of life concepts: a qualitative study. Lupus. 2010;19(14):1640-7.

18. Eyckmans L, Hilderson D, Westhovens R, Wouters C, Moons P. What does it mean to grow up with juvenile idiopathic arthritis? A qualitative study on the perspectives of patients. Clin Rheumatol. 2011;30(4):459-65.

19. Kristiansen TM, Primdahl J, Antoft R, Horslev-Petersen K. Everyday life with rheumatoid arthritis and implications for patient education and clinical practice: a focus group study. Musculoskeletal Care. 2012;10(1):29-38.

20. Meade T, Sharpe L, Hallab L, Aspanell D, Manolios N. Navigating motherhood choices in the context of rheumatoid arthritis: women's stories. Musculoskeletal Care. 2013:11(2):73-82

21. Feldthusen C, Bjork M, Forsblad-d'Elia H, Mannerkorpi K. Perception, consequences, communication, and strategies for handling fatigue in persons with rheumatoid arthritis of working age--a focus group study. Clin Rheumatol. 2013:32(5):557-66.

22. Nota I, Drossaert CHC, Taal E, van de Laar MAFJ. Patients' considerations in the decision-making process of initiating disease-modifying antirheumatic drugs. Arthritis Care Res. 2015;67(7):956-64.

23. Ammerlaan JW, van Os-Medendorp $\mathrm{H}$, de Boer-Nijhof $\mathrm{N}$, et al. Preferences and needs of patients with a rheumatic disease regarding the structure and content of online self-management support. Patient Educ Couns. 2017;100(3):501-8.

24. Feddersen $\mathrm{H}$, Mechlenborg Kristiansen T, Tanggaard Andersen P, HorslevPetersen K, Primdahl J. Juggling identities of rheumatoid arthritis, motherhood and paid work - a grounded theory study. Disabil Rehabil. 2019;41(13):1536-44.

25. Birru Talabi M, Clowse MEB, Blalock SJ, Hamm M, Borrero S. Perspectives of adult rheumatologists regarding family planning counseling and care: a qualitative study. Arthritis Care Res (Hoboken). 2020;72(3):452-8.

26. Clowse MEB, Eudy AM, Revels J, Neil L, Sanders GD. Provider perceptions on the management of lupus during pregnancy: barriers to improved care. Lupus. 2019;28(1):86-93.

27. Gomez AM, Arteaga S, Ingraham N, Arcara J. Medical conditions, pregnancy perspectives and contraceptive decision-making among young people: an exploratory, qualitative analysis. Contraception. 2019;100(1):72-8.

28. Phuti A, Schneider M, Makan K, Tikly M, Hodkinson B. Living with systemic lupus erythematosus in South Africa: a bitter pill to swallow. Health Qual Life Outcomes. 2019;17(1):65.

29. Chew C, Rebic N, Baldwin C, Amiri N, Proulx L, De Vera MA. "r/Thritis", pregnancy, and parenting: a qualitative descriptive study of Reddit forums to explore information needs and concerns of women with rheumatoid arthritis. ACR Open Rheumatol. 2019;1(8):485-92.

30. Phuti A, Hodkinson B, Tikly M, Schneider M. The feeling of not being entitled to something': fertility, pregnancy, and sexuality among women with systemic lupus erythematosus in South Africa. Scand J Rheumatol. 2020:49(3):214-20

31. Götestam Skorpen C, Hoeltzenbein M, Tincani A, et al. The EULAR points to consider for use of antirheumatic drugs before pregnancy, and during pregnancy and lactation. Ann Rheum Dis. 2016;75(5):795. 
32. Flint J, Panchal S, Hurrell A, et al. BSR and BHPR guideline on prescribing drugs in pregnancy and breastfeeding — part I: standard and biologic disease modifying anti-rheumatic drugs and corticosteroids. Rheumatology. 2016;55(9):1693-7.

33. Flint J, Panchal S, Hurrell A, et al. BSR and BHPR guideline on prescribing drugs in pregnancy and breastfeeding - part II: analgesics and other drugs used in rheumatology practice. Rheumatology. 2016;55(9):1698-702.

34. Sammaritano LR, Bermas BL, Chakravarty EE, et al. 2020 American College of Rheumatology guideline for the management of reproductive health in rheumatic and musculoskeletal diseases. Arthritis Rheumatol. 2020;72(4):529-56.

35. Doyle LH. Synthesis through meta-ethnography: paradoxes, enhancements, and possibilities. Qual Res. 2003;3(3):321-44.

\section{Publisher's Note}

Springer Nature remains neutral with regard to jurisdictional claims in published maps and institutional affiliations. 